\title{
MicroRNA-1207-5p inhibits hepatocellular carcinoma cell growth and invasion through the fatty acid synthase-mediated Akt/mTOR signalling pathway
}

\author{
GANG ZHAO, LEI DONG, HAITAO SHI, HONG LI, XIAOLAN LU, XIAOYAN GUO and JINHAI WANG \\ Department of Gastroenterology, The Second Affiliated Hospital of Xi'an Jiaotong University, \\ Xi'an, Shaanxi 710004, P.R. China
}

Received February 28, 2016; Accepted April 9, 2016

DOI: $10.3892 /$ or.2016.4952

\begin{abstract}
Fatty acid synthase (FASN) has emerged as a unique oncologic target for the treatment of cancers, including hepatocellular carcinoma (HCC). However, effective inhibitors of FASN for cancer treatment are lacking. MicroRNAs (miRNAs) have emerged as novel and endogenic inhibitors of gene expression. In the present study, we aimed to investigate the role of miR-1207-5p in HCC and the regulation of FASN through miR-1207-5p. The expression of miR-1207-5p was markedly reduced in HCC tissues and cell lines as detected with real-time quantitative polymerase chain reaction (qPCR). Overexpression of miR-1207-5p significantly suppressed the cell growth and invasion of HCC cells. By contrast, inhibition of miR-1207-5p exhibited an opposite effect. Bioinformatics analysis showed that FASN is a predicted target of miR-1207-5p which was validated by dual-luciferase reporter assay, qPCR and western blot analysis. Overexpression of miR-1207-5p inhibited the Akt/mTOR signalling pathway, and promotion of this pathway was noted following inhibition of miR-1207-5p. Rescue experiments showed that the restoration of FASN expression partially reversed the inhibitory effect of miR-1207-5p on cell growth, invasion and Akt phosphorylation. In conclusion, our study suggests that miR-1207-5p/FASN plays an important role in HCC, and provides novel insight into developing new inhibitors for FASN for therapeutic interventions for HCC.
\end{abstract}

Correspondence to: Dr Lei Dong, Department of Gastroenterology, The Second Affiliated Hospital of Xi'an Jiaotong University, 157 West 5th Road, Xi'an, Shaanxi 710004, P.R. China E-mail: donglei_xajt@163.com

Abbreviations: FASN, fatty acid synthase; HCC, hepatocellular carcinoma; miRNAs, microRNAs; qPCR, real-time quantitative polymerase chain reaction; UTR, untranslated region

Key words: fatty acid synthase, hepatocellular carcinoma, microRNAs, miR-1207-5p, Akt/mTOR

\section{Introduction}

Hepatocellular carcinoma (HCC) is a leading cause of cancer-related deaths worldwide (1). Despite current advancements in HCC treatment, the 5-year survival rate of HCC patients remains low (2). At present, an effective therapeutic method for HCC is lacking due to the obscure molecular mechanisms underlying the pathogenesis of HCC. The malignant growth, invasiveness and metastasis of HCC cells restrict the therapeutic effects of surgical resection, chemotherapy and radiofrequency ablation. Therefore, a deep understanding of the molecular mechanisms involved in the malignance and metastasis of cancer cells is helpful in developing novel therapies for HCC patients.

In recent years, an increasing number of studies have suggested that lipid metabolism which increases energy storage, membrane synthesis and signalling functions is essential for malignant cell growth and proliferation $(3,4)$. Fatty acid synthase (FASN) is an important enzyme for the synthesis of fatty acids and plays critical roles in lipid metabolism (5). Under normal conditions, FASN is usually silenced in many tissues without significant consequences (6). However, the overexpression of FASN has been determined in various types of cancers, including breast, ovarian, pancreatic, colorectal and prostate cancer, whereas the high expression of FASN is correlated with increasing tumor burden and poor patient prognosis (7-11). The overexpression of FASN promotes the cell growth and proliferation of cancer cells (12). Given the function of FASN in the modification of proteins through palmitoylation, FASN is associated with the activation of various oncogenic signalling pathways, including the Wnt/ $\beta$-catenin, protein kinase $\mathrm{C}$, human epithelial growth factor receptor 2 and Akt/mTOR pathways (13-15). Therefore, FASN represents a potential molecular target for inhibiting cancer. Indeed, various FASN inhibitors have been developed (16). However, few of these inhibitors have been tested in clinical trials due to their side-effects. Therefore, the development of novel inhibitors for FASN is important for cancer treatment.

MicroRNAs (miRNAs), which are small non-coding RNAs, have emerged as novel inhibitors of gene expression (17). miRNAs regulate gene expression by interacting with the 3'-untranslated region (UTR) of mRNAs causing 
translational inhibition (18). Therefore, miRNAs participate in various biological processes, including cell proliferation, invasion and metastasis, and they are involved in the pathogenesis of cancers (19). miRNAs can serve as potential biomarkers for cancer diagnosis and prognosis and potential molecular targets for cancer therapy $(20,21)$. Various miRNAs are involved in HCC (22,23). miR-1207-5p is a tumor-suppressive miRNA in gastric cancer that inhibits gastric cancer cell growth (24). However, whether miR-1207-5p plays an important role in $\mathrm{HCC}$ remains unexplored.

FASN is overexpressed in HCC tissues and cells, and knockdown of FASN impairs the apoptosis, proliferation, migration and invasion of HCC cells (25). A high expression level of FASN is also correlated with poor prognosis (26). However, the regulation of FASN by miRNAs remains poorly understood. In this study, we aimed to investigate the potential role of miR-1207-5p in HCC and its regulatory effect on FASN. We demonstrated that miR-1207-5p was significantly decreased in HCC clinical tissues and cancer cell lines, and the overexpression of miR-1207-5p inhibited HCC cell growth and invasion. We determined that FASN was a predicted target of miR-1207-5p. We also verified that miR-1207-5p could function through FASN to regulate HCC cell growth and invasion. In conclusion, our study indicated that miR-1207-5p is a potential and promising inhibitor of FASN in HCC.

\section{Materials and methods}

Cell culture. HCC cell lines, SMMC-7721, HepG2, MHCC97H and SK-HEP-1, and the normal liver cell line HL-7702 were obtained from the American Type Culture Collection (ATCC; Manassas, VA, USA). All these cells were grown in Dulbecco's modified Eagle's medium (DMEM) containing 10\% fetal bovine serum (FBS) and $100 \mathrm{U} / \mathrm{ml}$ penicillin/streptomycin mix (both from Gibco, Grand Island, NY, USA). The cells were cultured in a humidified incubator containing $5 \% \mathrm{CO}_{2}$ at $37^{\circ} \mathrm{C}$.

Patient specimens. Resected HCC samples from $20 \mathrm{HCC}$ patients and the matched adjacent normal liver tissues were provided by the Second Affiliated Hospital of Xi'an Jiaotong University with informed consent from the patients. The specimens were collected from HCC patients who underwent curative resection without prior chemotherapy or radiotherapy or other treatment procedures. The resected tissues were snap frozen in liquid nitrogen and stored at $-80^{\circ} \mathrm{C}$ for subsequent experiments. The experimental procedures were reviewed and approved by the Institutional Human Experiment and Ethics Committee of the Second Affiliated Hospital of Xi'an Jiaotong University.

RNA extraction and real-time quantitative polymerase chain reaction $(q P C R)$. The total RNA from the $\mathrm{HCC}$ tissues or $\mathrm{HCC}$ cells was extracted using the miRNeasy Mini kit and RNeasy Mini kit (Qiagen, Valencia, CA, USA) according to the manufacturer's instructions. Then, cDNA was synthesised with M-MLV Reverse Transcriptase (Invitrogen, Carlsbad, CA, USA) for mRNA detection or miScript Reverse Transcription kit (Qiagen) for miRNA detection. The cDNA templates were amplified using the SYBR-Green PCR kit (Qiagen). GAPDH and U6 were used as internal controls. The qPCR data were analysed using the $2^{-\Delta \Delta \mathrm{Ct}}$ method.

Cell transfection. The miR-1207-5p mimic, the miR-1207 inhibitor (anti-miR-1207-5p) and their negative controls (NCs) were obtained from RiboBio (Guangzhou, China). All miRNA oligonucleotides were transiently transfected into cells with Lipofectamine 2000 (Invitrogen) for a final concentration of $50 \mathrm{nM}$. The pCMV6-XL4/FASN recombinant vector carrying FASN cDNA without 3'-UTR and the miR-1207-5p mimic was co-transfected into the HCC cells with Lipofectamine 2000. After incubation for $48 \mathrm{~h}$, the transfection efficiency was detected using qPCR or western blot analysis.

Cell growth assay. Cell growth was detected using the 3-(4,5-dimethylthiazol-2-yl)-2,5-diphenyltetrazolium bromide (MTT) assay. The cells were briefly seeded into a 96-well plate and cultured for $48 \mathrm{~h}$ with the indicated treatments. Then, the cells were employed to measure cell growth by adding MTT solution ( $20 \mu \mathrm{l} / \mathrm{well}, 5 \mathrm{mg} / \mathrm{ml}$ in PBS; Sigma, St. Louis, MO, USA). The incubation was performed for $4 \mathrm{~h}$, and the formazan products were dissolved with dimethyl sulfoxide (200 $\mu \mathrm{l} /$ well). The absorbance value at $490 \mathrm{~nm}$ was read on a multiwell spectrophotometer (Bio-Tek Instruments, Winooski, VT, USA).

Transwell invasion assay. Cell invasion was detected using a Matrigel-coated Transwell (BD Biosciences, Franklin Lakes, NJ, USA). Briefly, $1 \times 10^{5}$ cells transfected with the miR-1207-5p mimic or anti-miR-1207-5p in $200 \mu \mathrm{l}$ of serum-free medium were plated into the top chamber, whereas the bottom chamber was filled with medium containing $10 \%$ FBS. The cells were incubated for $24 \mathrm{~h}$. Then, the non-invasive cells were removed from the top chamber. The invaded cells on the bottom chamber were fixed and stained with crystal violet $(0.05 \%)$. The stained cells were observed and counted under a microscope. The number of invasive cells from five random fields in the same slide was counted and averaged.

Western blot analysis. The protein was separated with SDS-polyacrylamide gels, and the separated protein was then transferred onto a nitrocellulose membrane (Bio-Rad,Hercules, CA, USA). The membrane was blocked and incubated with primary antibodies at $4^{\circ} \mathrm{C}$ overnight. Then, the membrane was incubated with horseradish peroxidase-conjugated secondary antibody (Santa Cruz Biotechnology, Inc., Santa Cruz, CA, USA) for $1 \mathrm{~h}$ at $37^{\circ} \mathrm{C}$. The protein bands on the membrane were visualised through an enhanced chemiluminescence method. The primary antibodies, including rabbit anti-human FASN and GAPDH antibodies, were purchased from Santa Cruz Biotechnology, Inc. The rabbit anti-human mTOR and phosphorylated mTOR (p-mTOR) antibodies were obtained from Abcam (Cambridge, MA, USA). The rabbit anti-human Akt and p-Akt antibodies were purchased from Cell Signaling Technology (Danvers, MA, USA).

Dual-luciferase reporter assay. The cDNA fragment of FASN 3'-UTR containing the predicted binding sites of miR-1207-5p or the mutated sequence was inserted into the pmirGLO luciferase reporter vector (Promega, Madison, WI, USA). The 

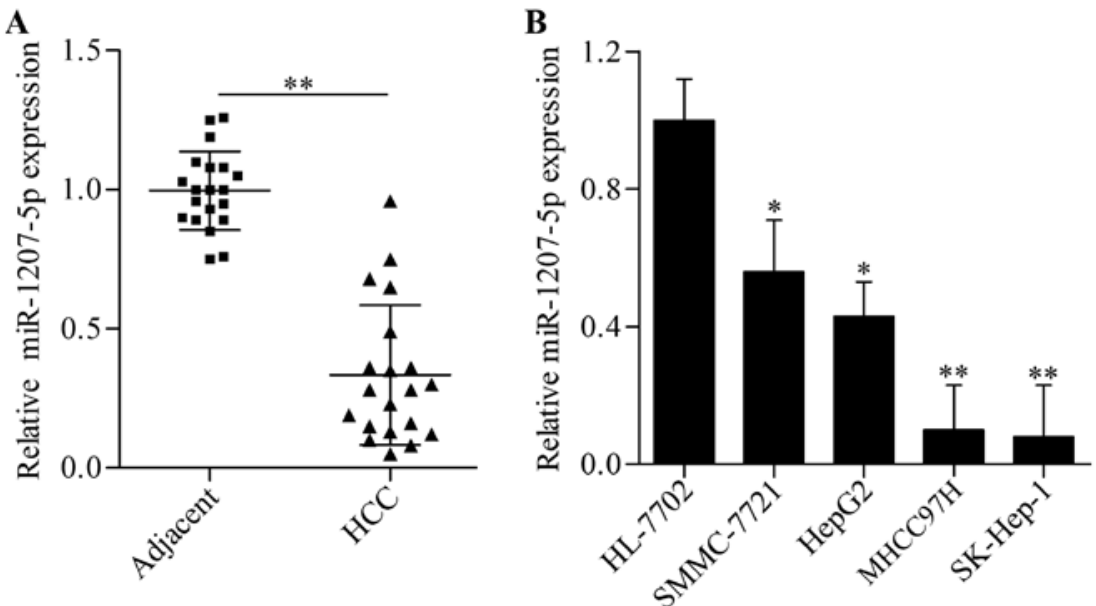

Figure 1. Decreased expression of miR-1207-5p in the HCC tissues and cell lines. (A) Analysis of of miR-1207-5p expression in 20 pairs of human HCC tissues and the pair-matched adjacent non-tumorous tissues detected via qPCR analysis. ${ }^{* *} \mathrm{P}<0.01$. (B) Analysis of miR-1207 expression in HCC cell lines (SMMC-7721, HepG2, MHCC97H and SK-HEP-1) and normal liver cell line HL-7702 via qPCR. HL-7702 was used as the control. "P<0.05 and "p $<0.01$ vs. HL-7702. HCC, hepatocellular carcinoma; qPCR, real-time quantitative polymerase chain reaction.

recombinant reporter vectors and the miR-1207-5p mimic or anti-miR-1207-5p were co-transfected into the SK-HEP-1 cells and incubated for $48 \mathrm{~h}$. The luciferase activity was detected using the Dual-Luciferase system kit (Promega). The data are represented as firefly/Renilla luciferase activity.

Data analysis. The data are expressed as means \pm standard deviation and were analysed using SPSS 15.0 software package (SPSS, Inc., Chicago, IL, USA). The statistical significance between the two groups was analysed with Student's t-test, whereas multiple comparison was detected through one-way analysis of variance followed by Bonferroni test. The differences were regarded as statistically significant at $\mathrm{p}<0.05$.

\section{Results}

miR-1207-5p is downregulated in HCC tissues and cell lines. To investigate whether miR-1207 plays an important role in HCC, we first analysed the expression profile of miR-1207-5p in 20 pairs of human HCC tissues and pair-matched adjacent non-tumorous tissues via qPCR analysis. The results showed that miR-1207 was significantly downregulated in the HCC tissues compared with that in the adjacent non-tumorous tissues (Fig. 1A). To further verify the lower expression level of miR-1207-5p in HCC, we detected the expression level of miR-1207-5p in HCC cell lines, including SMMC-7721, HepG2, MHCC97H and SK-HEP-1. We also found a significant decrease in miR-1207-5p in the HCC cell lines as compared with that in the normal liver cell line HL-7702 (Fig. 1B). These results indicate that miR-1207-5p plays an important role in the development and progression of HCC.

Overexpression of miR-1207-5p suppresses HCC cell growth and invasion. To investigate the potential function of miR-1207-5p in HCC, we ascertained whether miR-1207-5p affects the cell growth and invasion of HCC in vitro. For this purpose, we performed gain-of-function and loss-of-function experiments via transfection of the miR-1207-5p mimic or anti-miR-1207-5p into the MHCC97H and SK-HEP-1 cells.
MTT assay showed that the overexpression of miR-1207-5p via transfection with the miR-1207-5p mimic significantly decreased the cell growth and viability of the MHCC97H (Fig. 2A) and SK-HEP-1 (Fig. 2B) cells, whereas the depletion of miR-1207-5p via anti-miR-1207-5p displayed an opposite effect. The overexpression of miR-1207-5p significantly inhibited HCC cell invasion. In contrast, cell invasion was markedly increased with the depletion of miR-1207-5p (Fig. 2C and D). The data implied that miR-1207-5p inhibited HCC cell growth and invasion.

FASN is a target of miR-1207-5p. To investigate the molecular mechanisms of miR-1207-5p in regulating HCC cell growth and invasion, we analysed the putative target genes of miR-1207-5p. Interestingly, we determined that FASN, an important target gene for cancer treatment (5), was the predicted target of miR-1207-5p (Fig. 3A). To determine whether miR-1207-5p directly targets the 3'-UTR of FASN, we performed a dual-luciferase reporter assay. Luciferase reporter vector containing FASN 3'-UTR with the seed-region for miR-1207-5p was constructed and transfected into SK-HEP-1 cells in the presence of the miR-1207-5p mimic or anti-miR-1207-5p. The results showed that transfection with the miR-1207-5p mimic significantly inhibited the luciferase activity, whereas depletion of miR-1207-5p markedly promoted the luciferase activity of the luciferase reporter (Fig. 3B). To further verify the specificity of this effect, the seed-region of the miR-1207-5p binding site on the 3'-UTR of FASN was mutated. The results demonstrated that neither miR-1207-5p overexpression nor depletion had an obvious effect on the mutated luciferase reporter vector (Fig. 3B). To determine whether miR-1207-5p regulates the expression of FASN, we transfected SK-HEP-1 cells with the miR-1207-5p mimic or anti-miR-1207-5p and then detected FASN expression. The results showed that both mRNA (Fig. 3C) and the protein (Fig. 3D) expression of FASN were significantly decreased via miR-1207-5p overexpression or markedly increased via miR-1207-5p depletion. Similar data were observed with the MHCC97H cells (data not shown). Altogether, these results revealed that miR-1207-5p regulated FASN expression by binding to its 3'-UTR. 

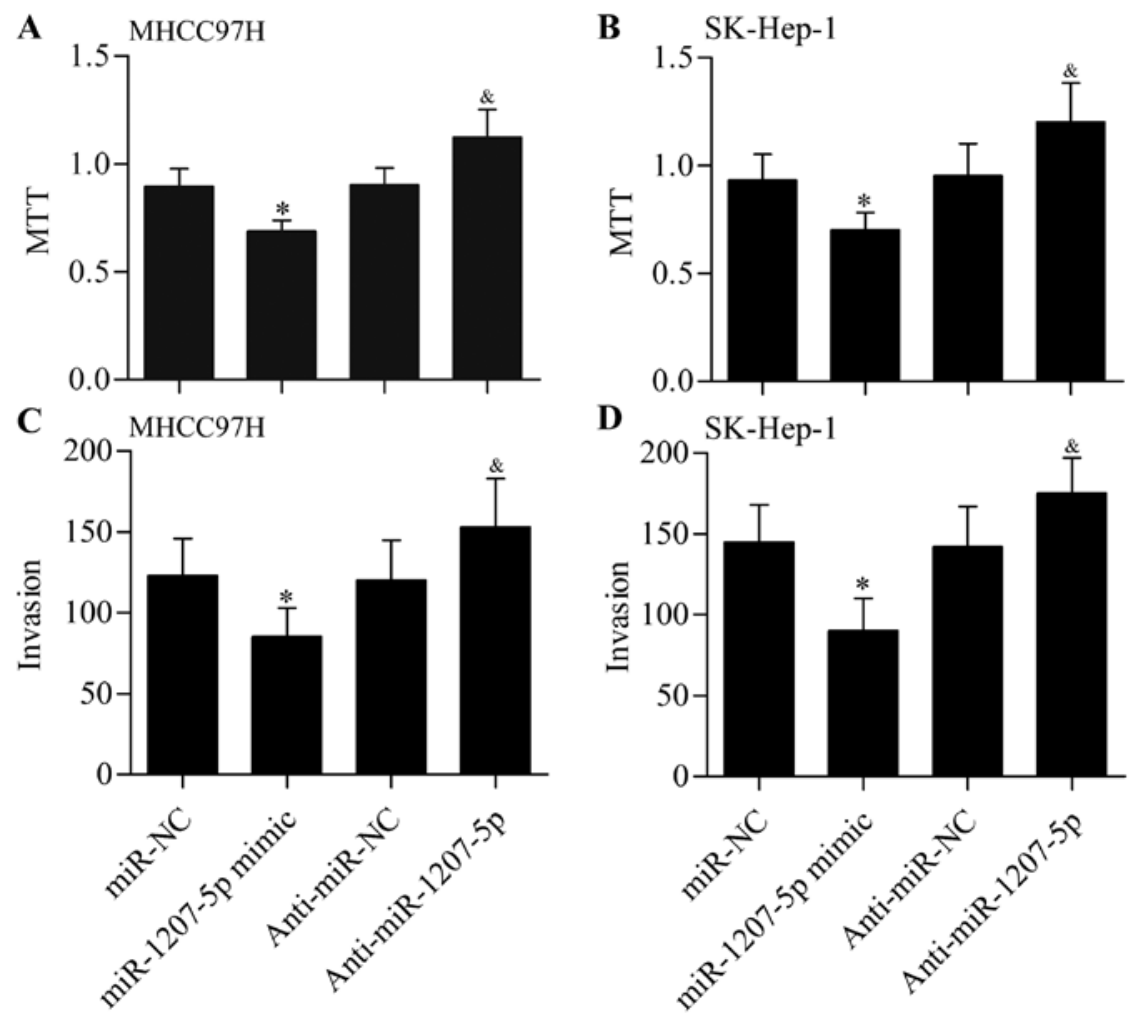

Figure 2. miR-1207-5p regulates the cell growth and invasion of HCC cells. The effect of miR-1207-5p overexpression or depletion in (A) MHCC97H and (B) SK-HEP-1 cells was detected using the MTT assay. The cells were transfected with the miR-1207-5p mimic or anti-miR-1207-5p for $48 \mathrm{~h}$ and then subjected to the MTT assay. The effect of miR-1207-5p overexpression or depletion on cell invasion was detected by Transwell invasion assay with (C) MHCC97H and (D) SK-HEP-1 cells. "P<0.05 vs. miR-NC; ${ }^{\text {p }}$ < 0.05 vs. anti-miR-NC. HCC, hepatocellular carcinoma; NC, negative control.

A
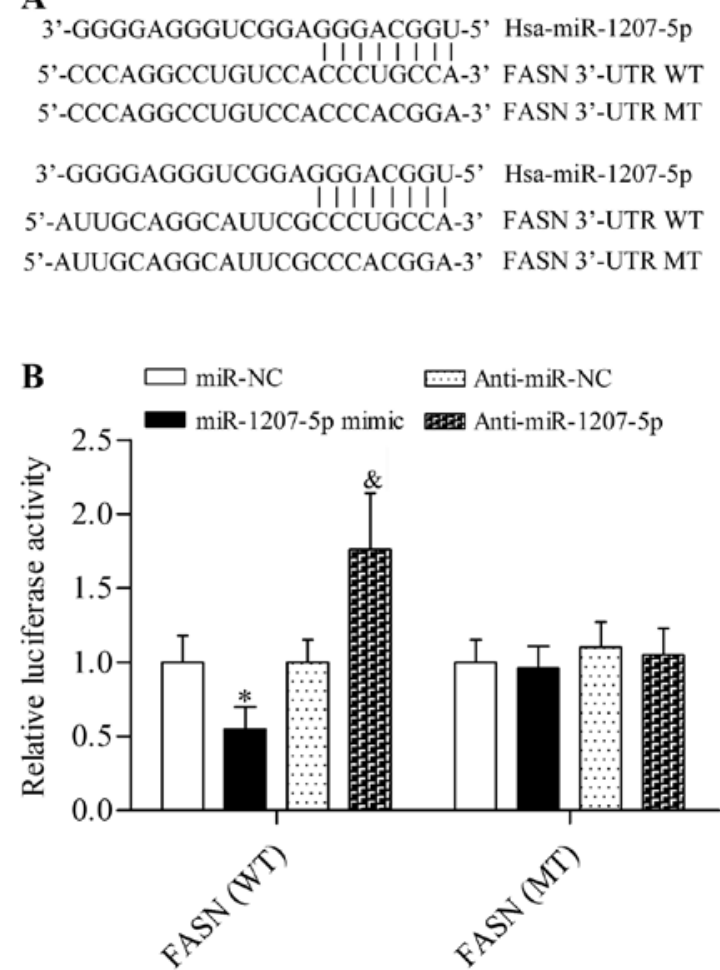

C
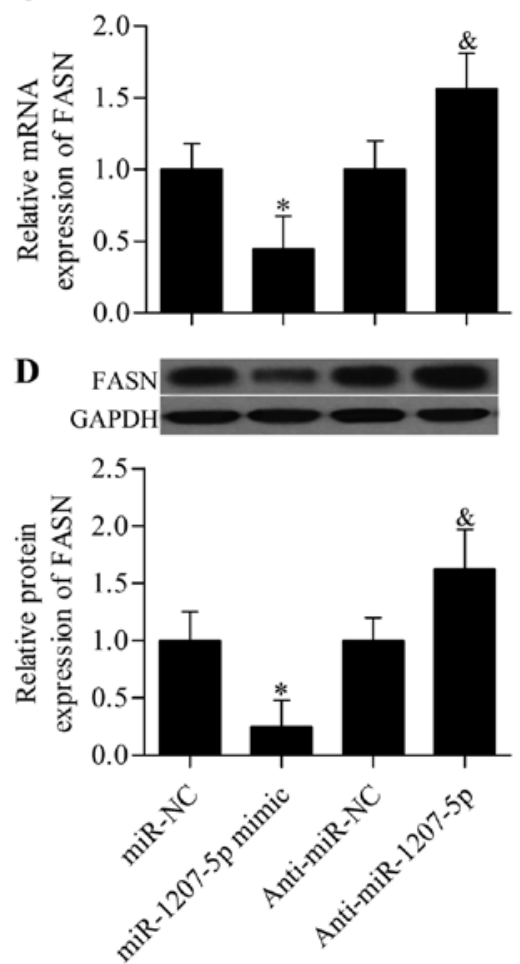

Figure 3. miR-1207-5p regulates FASN expression. (A) The seed-region of the miR-1207-5p binding site on the 3'-UTR of FASN. (B) Dual-luciferase reporter assay for miR-1207-5p and FASN 3'-UTR. SK-HEP-1 cells were transfected with the WT or the MT of the FASN 3'-UTR vector together with the miR-1207-5p mimic or anti-miR-1207-5p. After $48 \mathrm{~h}$, the luciferase activity was detected. The effect of miR-1207-5p overexpression or depletion on (C) mRNA and (D) protein expression of FASN in the SK-HEP-1 cells was detected by qPCR and western blot analysis, respectively. Cells were transfected with the miR-1207-5p mimic or anti-miR-1207-5p and incubated for $48 \mathrm{~h}$ before being harvested for qPCR or western blot analysis. " $\mathrm{P}<0.05 \mathrm{vs}$. miR-NC; ${ }^{\&} \mathrm{p}<0.05 \mathrm{vs}$. anti-miR-NC. FASN, fatty acid synthase; UTR, untranslated region; WT, wild-type; MT, mutated type; qPCR, real-time quantitative polymerase chain reaction; NC, negative control. 
A
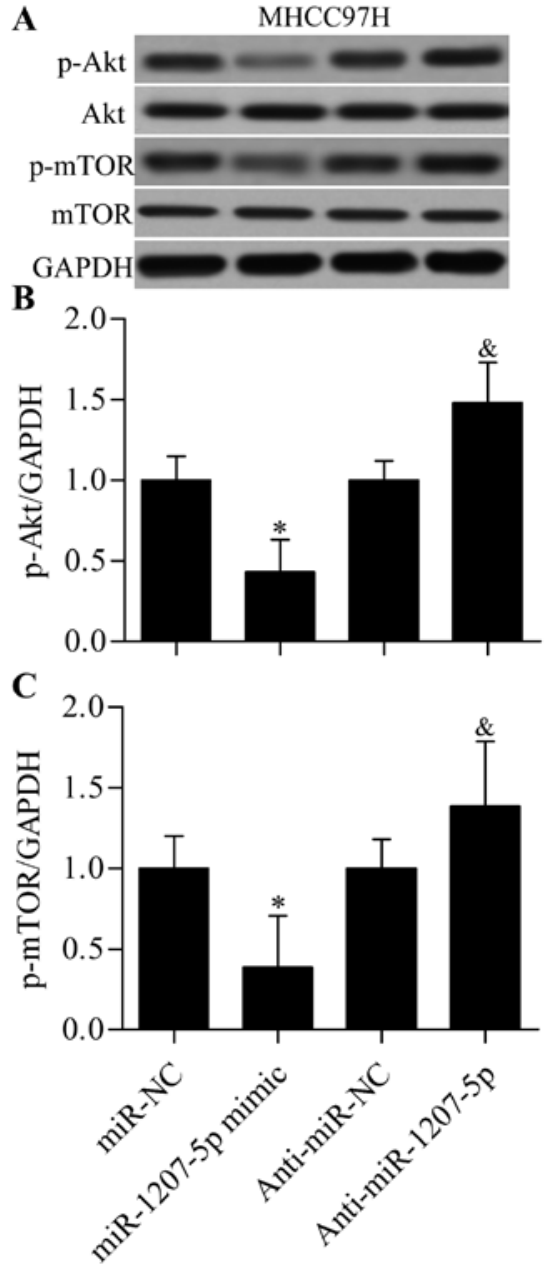

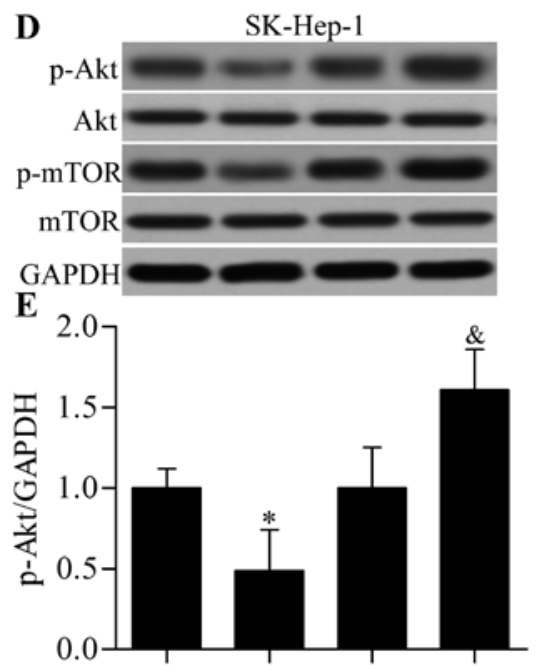

F

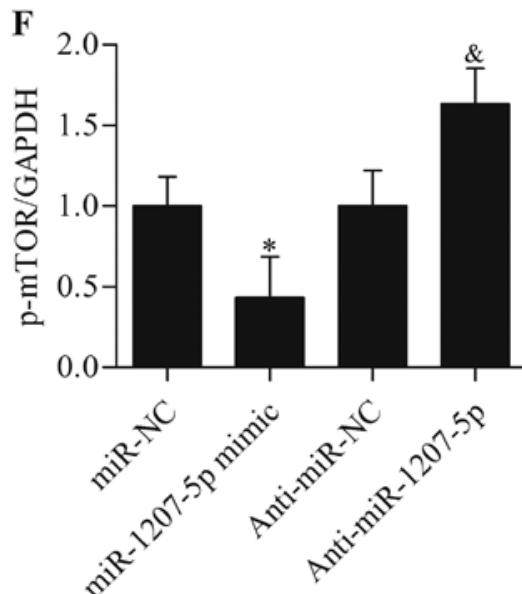

Figure 4. Effect of miR-1207-5p on the Akt/mTOR signalling pathway. Effect of miR-1207-5p overexpression or depletion on the protein expression of Akt, p-Akt, mTOR and phosphorylated (p)-mTOR in (A-C) MHCC97H and (D-F) SK-HEP-1 cells was detected by western blot analysis. * $<0.05$ vs. miR-NC; ${ }^{\&} \mathrm{p}<0.05$ vs. anti-miR-NC. NC, negative control.

Overexpression of miR-1207-5p inhibits the Akt/mTOR signalling pathway. FASN has been reported as an important regulator of various oncogenic signalling pathways (5). Due to the regulatory effect of miR-1207-5p on FASN expression, we sought to detect the effect of miR-1207-5p on the Akt/mTOR signalling pathway which plays an important role in the development and progression of $\operatorname{NSCLC}(27,28)$. Here, we found that miR-1207-5p overexpression significantly reduced the protein expression levels of $\mathrm{p}$-Akt and $\mathrm{p}$-mTOR in the MHCC97H (Fig. 4A-C) and SK-HEP-1 cells (Fig. 4D-F) whereas depletion of miR-1207-5p markedly promoted the expression levels of $\mathrm{p}-\mathrm{Akt}$ and $\mathrm{p}-\mathrm{mTOR}$. The results suggest that miR-1207-5p is an important regulator of the Akt/mTOR signalling pathway.

Restoration of FASN expression reverses the effect of the miR-1207-5p mimic. To validate whether miR-1207-5p functions through FASN, we transfected cells with the FASN expression vector and the miR-1207-5p mimic. The results showed that FASN expression vector transfection significantly restored the FASN protein expression, which was decreased by miR-1207-5p overexpression (Fig. 5A and B). Interestingly, the decreased expression of $\mathrm{p}$-Akt induced by miR-1207-5p overexpression was partially reversed by FASN restoration (Fig. 5A and B).
Furthermore, the inhibitory effect of miR-1207 overexpression on HCC cell growth (Fig. 5C and D) and invasion (Fig. 5E and F) was also significantly abolished by FASN restoration. Overall, our results indicated that miR-1207-5p functions in HCC cells by directly regulating FASN.

\section{Discussion}

In the present study, our results indicated that the expression of miR-1207-5p was significantly reduced in the HCC tissues and cell lines. The overexpression of miR-1207-5p inhibited HCC cell growth and invasion. Hence, our study for the first time revealed that miR-1207-5p is a tumor suppressor in the development and progression of HCC. However, the precise molecular mechanism of miR-1207-5p in HCC remains to be determined.

In gastric cancer, patients with high expression of miR-1207-5p have slight lymphovascular invasion and a good prognosis compared with patients with low expression of miR-1207-5p (29). Chen et al revealed that miR-1207-5p is significantly downregulated in gastric tumor samples, and the overexpression of miR-1207-5p inhibits cell growth and invasion of gastric cancer cells by targeting human telomerase reverse transcriptase (hTERT) (24). A recent study demonstrated that 

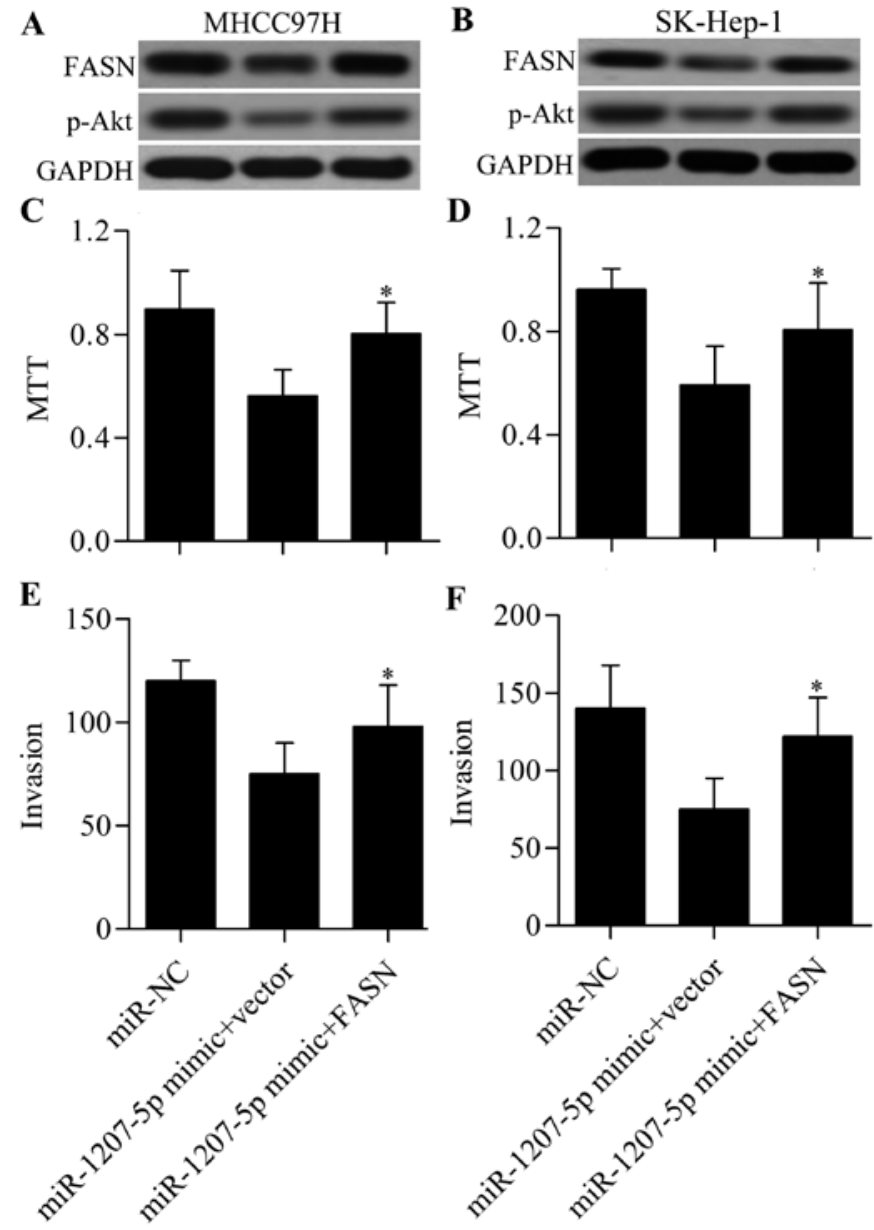

Figure 5. miR-1207-5p functions through FASN in HCC cells. Western blot analysis of FASN and p-Akt protein expression in (A) $\mathrm{MHCC} 97 \mathrm{H}$ and (B) SK-HEP-1 cells. The cells were co-transfected with miR-1207-5p mimic and pCMV6-XL4/FASN carrying full-length FASN cDNA without the 3'-UTR region and were incubated for $48 \mathrm{~h}$. The effect of FASN restoration on cell growth of (C) MHCC97H and (D) SK-HEP-1 cells was detected with MTT assay. The effect of FASN restoration on cell invasion of (E) MHCC97H and (F) SK-HEP-1 cells was detected with Transwell invasion assay. ${ }^{*} \mathrm{P}<0.05$ vs. $\mathrm{miR}-1207-5 \mathrm{p}+$ vector. FASN, fatty acid synthase; HCC, hepatocellular carcinoma; UTR, untranslated region.

the long non-coding RNA BC032469 increased gastric cancer cell proliferation by impairing miR-1207-5p-dependent hTERT downregulation (30). Moreover, miR-1207-5p was also found to inhibit epithelial-mesenchymal transition of nasopharyngeal cancers induced by growth factors (31). miR-1207-5p also plays an important role in breast and non-small cell lung cancers $(32,33)$. However, the role of miR-1207-5p in HCC has not been well studied. Our study demonstrated that miR-1207-5p is downregulated in HCC and miR-1207-5p also functions as a tumor-suppressive miRNA that inhibits cell proliferation and invasion, at least in part, by targeting FASN.

FASN is an important gene in the regulation of lipid metabolism (5). Evidence indicates an oncogenic role of FASN in various cancers (34). Given that the liver is an important organ for lipid metabolism, the dysregulation of FASN plays an important role in HCC. FASN is highly expressed in HCC tissues and cancer cell lines and knockdown of FASN inhibits cell proliferation, invasion and migration of HCC cells (25). FASN overexpression combined with loss of phosphatase and tensin homolog is correlated with worse prognosis in Chinese patients with HCC (26). Inhibition of lipogenesis by targeting FASN significantly suppressed the tumor growth of HCC (35-37). Inhibition of FASN also resensitised HCC cells to taxane (38). Therefore, FASN represents a promising molecular target for HCC. Various FASN inhibitors have been developed in recent years $(16,39)$. However, the majority of these inhibitors have not been applied in clinical trials due to their side-effects (5). In the present study, we determined that miR-1207-5p was a novel inhibitor of FASN by targeting the 3'-UTR of FASN. We determined that overexpression of miR-1207-5p significantly decreased the expression of FASN in HCC cells, providing novel insights into developing novel FASN inhibitors.

The emerging role of miRNAs in lipid metabolism has been highlighted in recent years (40). FASN can be regulated by specific miRNAs. In triple-negative breast cancer cells, FASN is a functional target of miR-193b, which is involved in metformin-induced cytotoxicity (41). Yang et al found that miRNA-142-3p inhibited osteosarcoma cell proliferation by suppressing FASN expression (42). Similarly, Cheng et al reported that miR-320 inhibited osteosarcoma cell proliferation by targeting FASN (43). The overexpression of miR-107 induced endoplasmic reticulum stress and lipid accumulation in hepatocytes by directly inhibiting FASN (44). In the present study, our results indicated that miR-1207-5p can directly target and inhibit FASN in HCC cells. Our findings as well as these aforementioned reports support the notion that miRNAs can serve as novel inhibitors of FASN and that they can be used as potential therapeutic tools for HCC treatment.

FASN modulates receptor localisation through palmitoylation and promotes the activation of oncogenic signalling pathways, such as the Akt/mTOR signalling pathway (5). The Akt/mTOR signalling pathway also promotes the transcription of FASN, thereby forming a positive feedback regulation $(45,46)$. The downregulation of FASN inhibited cancer cell invasion and migration by targeting the Akt/mTOR signalling pathway (47-49). A recent study reported that inactivation of FASN suppressed Akt-driven hepatocarcinogenesis in mice and humans (50). In line with these findings, we revealed that inhibition of FASN with miR-1207-5p significantly impeded the Akt/mTOR signalling pathway in HCC. Restoration of FASN reversed the inhibitory effect of miR-1207-5p, thereby indicating that miR-1207-5p regulates the Akt/mTOR signalling pathway by directly regulating FASN.

Our study demonstrated that miR-1207-5p inhibits HCC cell growth and invasion by directly targeting and inhibiting FASN. For the first time, our results indicated the important role of miR-1207-5p in HCC and verified that miR-1207-5p functions as an endogenic inhibitor of FASN. Our study suggests that miR-1207-5p/FASN plays an important role in HCC, and provides novel insight into the development of novel inhibitors of FASN for cancer therapy.

\section{Acknowledgements}

This study was supported by the Natural Science Foundation of Shaanxi Province (no. 2012JQ4030) and the Talents Research Fund of the Second Affiliated Hospital of Xi'an Jiaotong University [RC(BL)201301]. 


\section{References}

1. Siegel RL, Miller KD and Jemal A: Cancer statistics, 2015. CA Cancer J Clin 65: 5-29, 2015.

2. Katagiri S and Yamamoto M: Multidisciplinary treatments for hepatocellular carcinoma with major portal vein tumor thrombus. Surg Today 44: 219-226, 2014.

3. Santos CR and Schulze A: Lipid metabolism in cancer. FEBS J 279: 2610-2623, 2012.

4. Tania M, Khan MA and Song Y: Association of lipid metabolism with ovarian cancer. Curr Oncol 17: 6-11, 2010.

5. Jones SF and Infante JR: Molecular pathways: Fatty acid synthase. Clin Cancer Res 21: 5434-5438, 2015.

6. Chakravarthy MV, Pan Z, Zhu Y, Tordjman K, Schneider JG, Coleman T, Turk J and Semenkovich CF: 'New' hepatic fat activates PPARalpha to maintain glucose, lipid, and cholesterol homeostasis. Cell Metab 1: 309-322, 2005.

7. Kuhajda FP, Jenner K, Wood FD, Hennigar RA, Jacobs LB, Dick JD and Pasternack GR: Fatty acid synthesis: A potential selective target for antineoplastic therapy. Proc Natl Acad Sci USA 91: 6379-6383, 1994.

8. Walter K, Hong SM, Nyhan S, Canto M, Fedarko N, Klein A, Griffith M, Omura N, Medghalchi S, Kuhajda F, et al: Serum fatty acid synthase as a marker of pancreatic neoplasia. Cancer Epidemiol Biomarkers Prev 18: 2380-2385, 2009.

9. Witkiewicz AK, Nguyen KH, Dasgupta A, Kennedy EP, Yeo CJ, Lisanti MP and Brody JR: Co-expression of fatty acid synthase and caveolin-1 in pancreatic ductal adenocarcinoma: Implications for tumor progression and clinical outcome. Cell Cycle 7: 3021-3025, 2008.

10. Long QQ, Yi YX, Qiu J, Xu CJ and Huang PL: Fatty acid synthase (FASN) levels in serum of colorectal cancer patients: Correlation with clinical outcomes. Tumour Biol 35: 3855-3859, 2014.

11. Cai Y, Wang J, Zhang L, Wu D, Yu D, Tian X, Liu J, Jiang X, Shen Y, Zhang L, et al: Expressions of fatty acid synthase and HER 2 are correlated with poor prognosis of ovarian cancer. Med Oncol 32: 391, 2015.

12. Vazquez-Martin A, Colomer R, Brunet J, Lupu R and Menendez JA: Overexpression of fatty acid synthase gene activates HER1/HER2 tyrosine kinase receptors in human breast epithelial cells. Cell Prolif 41: 59-85, 2008.

13. Fiorentino M, Zadra G, Palescandolo E, Fedele G, Bailey D, Fiore C, Nguyen PL, Migita T, Zamponi R, Di Vizio D, et al: Overexpression of fatty acid synthase is associated with palmitoylation of Wnt1 and cytoplasmic stabilization of beta-catenin in prostate cancer. Lab Invest 88: 1340-1348, 2008.

14. Benjamin DI, Li DS, Lowe W, Heuer T, Kemble G and Nomura DK: Diacylglycerol metabolism and signaling is a driving force underlying FASN inhibitor sensitivity in cancer cells. ACS Chem Biol 10: 1616-1623, 2015.

15. Menendez JA: Fine-tuning the lipogenic/lipolytic balance to optimize the metabolic requirements of cancer cell growth: Molecular mechanisms and therapeutic perspectives. Biochim Biophys Acta 1801: 381-391, 2010.

16. Zhou W, Simpson PJ, McFadden JM, Townsend CA, Medghalchi SM, Vadlamudi A, Pinn ML, Ronnett GV and Kuhajda FP: Fatty acid synthase inhibition triggers apoptosis during S phase in human cancer cells. Cancer Res 63: 7330-7337, 2003.

17. Winter J, Jung S, Keller S, Gregory RI and Diederichs S: Many roads to maturity: MicroRNA biogenesis pathways and their regulation. Nat Cell Biol 11: 228-234, 2009.

18. Bartel DP: MicroRNAs: Genomics, biogenesis, mechanism, and function. Cell 116: 281-297, 2004.

19. Lin S and Gregory RI: MicroRNA biogenesis pathways in cancer Nat Rev Cancer 15: 321-333, 2015.

20. Pichler M and Calin GA: MicroRNAs in cancer: From developmental genes in worms to their clinical application in patients. $\mathrm{Br}$ J Cancer 113: 569-573, 2015.

21. Hayes J, Peruzzi PP and Lawler S: MicroRNAs in cancer: Biomarkers, functions and therapy. Trends Mol Med 20: 460-469, 2014.

22. Li X, Yang W, Lou L, Chen Y, Wu S and Ding G: microRNA: A promising diagnostic biomarker and therapeutic target for hepatocellular carcinoma. Dig Dis Sci 59: 1099-1107, 2014.

23. Chang-Hao Tsao S, Behren A, Cebon J and Christophi C: The role of circulating microRNA in hepatocellular carcinoma. Front Biosci (Landmark Ed) 20: 78-104, 2015.
24. Chen L, Lü MH, Zhang D, Hao NB, Fan YH, Wu YY, Wang SM, Xie R, Fang DC, Zhang H, et al: miR-1207-5p and miR-1266 suppress gastric cancer growth and invasion by targeting telomerase reverse transcriptase. Cell Death Dis 5: e1034, 2014.

25. Hao Q, Li T, Zhang X, Gao P, Qiao P, Li S and Geng Z: Expression and roles of fatty acid synthase in hepatocellular carcinoma. Oncol Rep 32: 2471-2476, 2014.

26. Zhu X, Qin X, Fei M, Hou W, Greshock J, Bachman KE Wooster R, Kang J and Qin CY: Combined phosphatase and tensin homolog (PTEN) loss and fatty acid synthase (FAS) overexpression worsens the prognosis of Chinese patients with hepatocellular carcinoma. Int J Mol Sci 13: 9980-9991, 2012.

27. Gu Y, Körbel C, Scheuer C, Nenicu A, Menger MD and Laschke MW: Tubeimoside-1 suppresses tumor angiogenesis by stimulation of proteasomal VEGFR 2 and Tie 2 degradation in a non-small cell lung cancer xenograft model. Oncotarget 7 : 5258-5272, 2016

28. Li H, Hu J, Wu S, Wang L, Cao X, Zhang X, Dai B, Cao M, Shao R, Zhang R, et al: Auranofin-mediated inhibition of $\mathrm{PI} 3 \mathrm{~K} / \mathrm{AKT} / \mathrm{mTOR}$ axis and anticancer activity in non-small cell lung cancer cells. Oncotarget 7: 3548-3558, 2016.

29. Huang KH, Lan YT, Fang WL, Chen JH, Lo SS, Li AF, Chiou SH, Wu CW and Shyr YM: The correlation between miRNA and lymph node metastasis in gastric cancer. Biomed Res Int 2015: 543163,2015

30. Lü MH, Tang B, Zeng S, Hu CJ, Xie R, Wu YY, Wang SM He FT and Yang SM: Long noncoding RNA BC032469, a novel competing endogenous RNA, upregulates hTERT expression by sponging miR-1207-5p and promotes proliferation in gastric cancer. Oncogene: Nov 9, 2015 (Epub ahead of print).

31. Qin Z, He W, Tang J, Ye Q, Dang W, Lu Y, Wang J, Li G, Yan Q and Ma J: MicroRNAs provide feedback regulation of epithelial-mesenchymal transition induced by growth factors. J Cell Physiol 231: 120-129, 2016.

32. Ma R, Wang $\mathrm{C}$, Wang J, Wang $\mathrm{D}$ and $\mathrm{Xu} \mathrm{J}$ : miRNA-mRNA interaction network in non-small cell lung cancer. Interdiscip Sci: Apr 11, 2015 (Epub ahead of print).

33. Gonul O, Aydin HH, Kalmis E, Kayalar H, Ozkaya AB, Atay S and Ak H: Effects of Ganoderma lucidum (higher Basidiomycetes) extracts on the miRNA profile and telomerase activity of the MCF-7 breast cancer cell line. Int J Med Mushrooms 17: 231-239, 2015.

34. Flavin R, Peluso S, Nguyen PL and Loda M: Fatty acid synthase as a potential therapeutic target in cancer. Future Oncol 6: 551-562, 2010.

35. Bhalla K, Hwang BJ, Dewi RE, Ou L, Twaddel W, Fang HB, Vafai SB, Vazquez F, Puigserver P, Boros L, et al: PGC1a promotes tumor growth by inducing gene expression programs supporting lipogenesis. Cancer Res 71: 6888-6898, 2011.

36. Zhang H, Feng Z, Huang R, Xia Z, Xiang G and Zhang J: MicroRNA-449 suppresses proliferation of hepatoma cell lines through blockade lipid metabolic pathway related to SIRT1. Int J Oncol 45: 2143-2152, 2014.

37. Huang CH, Tsai SJ, Wang YJ, Pan MH, Kao JY and Way TD: EGCG inhibits protein synthesis, lipogenesis, and cell cycle progression through activation of AMPK in p53 positive and negative human hepatoma cells. Mol Nutr Food Res 53: 1156-1165, 2009.

38. Meena AS, Sharma A, Kumari R, Mohammad N, Singh SV and Bhat MK: Inherent and acquired resistance to paclitaxel in hepatocellular carcinoma: Molecular events involved. PLoS One 8: e61524, 2013

39. Hardwicke MA, Rendina AR, Williams SP, Moore ML, Wang L, Krueger JA, Plant RN, Totoritis RD, Zhang G, Briand J, et al: A human fatty acid synthase inhibitor binds $\beta$-ketoacyl reductase in the keto-substrate site. Nat Chem Biol 10: 774-779, 2014.

40. Yang Z, Cappello T and Wang L: Emerging role of microRNAs in lipid metabolism. Acta Pharm Sin B 5: 145-150, 2015.

41. Wahdan-Alaswad RS, Cochrane DR, Spoelstra NS, Howe EN, Edgerton SM, Anderson SM, Thor AD and Richer JK: Metformin-induced killing of triple-negative breast cancer cells is mediated by reduction in fatty acid synthase via miRNA-193b. Horm Cancer 5: 374-389, 2014

42. Yang YQ, Qi J, Xu JQ and Hao P: MicroRNA-142-3p, a novel target of tumor suppressor menin, inhibits osteosarcoma cell proliferation by down-regulation of FASN. Tumour Biol 35: 10287-10293, 2014.

43. Cheng C, Chen ZQ and Shi XT: MicroRNA-320 inhibits osteosarcoma cells proliferation by directly targeting fatty acid synthase. Tumour Biol 35: 4177-4183, 2014. 
44. Bhatia H, Verma G and Datta M: miR-107 orchestrates ER stress induction and lipid accumulation by post-transcriptional regulation of fatty acid synthase in hepatocytes. Biochim Biophys Acta 1839: 334-343, 2014.

45. Wang H, Luo QF, Peng AF, Long XH, Wang TF, Liu ZL, Zhang GM, Zhou RP, Gao S, Zhou Y, et al: Positive feedback regulation between Akt phosphorylation and fatty acid synthase expression in osteosarcoma. Int J Mol Med 33: 633-639, 2014.

46. Li J, Huang Q, Long X, Zhang J, Huang X, Aa J, Yang H, Chen Z and Xing J: CD147 reprograms fatty acid metabolism in hepatocellular carcinoma cells through Akt/mTOR/SREBP1c and P38/PPAR $\alpha$ pathways. J Hepatol 63: 1378-1389, 2015.

47. Wang TF, Wang H, Peng AF, Luo QF, Liu ZL, Zhou RP, Gao S, Zhou Y and Chen WZ: Inhibition of fatty acid synthase suppresses U-2 OS cell invasion and migration via downregulating the activity of HER2/PI3K/AKT signaling pathway in vitro. Biochem Biophys Res Commun 440: 229-234, 2013.
48. Zheng SS, Gao JG, Liu ZJ, Zhang XH, Wu S, Weng BW, Wang YL, Hou SC and Jiang B: Downregulation of fatty acid synthase complex suppresses cell migration by targeting phospho-AKT in bladder cancer. Mol Med Rep 13: 1845-1850, 2016.

49. Chang L, Wu P, Senthilkumar R, Tian X, Liu H, Shen X, Tao Z and Huang P: Loss of fatty acid synthase suppresses the malignant phenotype of colorectal cancer cells by down-regulating energy metabolism and mTOR signaling pathway. J Cancer Res Clin Oncol 142: 59-72, 2016.

50. Li L, Pilo GM, Li X, Cigliano A, Latte G, Che L, Joseph C, Mela M, Wang C, Jiang L, et al: Inactivation of fatty acid synthase impairs hepatocarcinogenesis driven by AKT in mice and humans. J Hepatol 64: 333-341, 2016. 